\title{
Rotation Frequency of Human Bronchial and Nasal Epithelial Spheroids as an Indicator of Mucociliary Function
}

\author{
Fernando Gamarra Albrecht Bergner Elisabeth Stauss Ingrid Stocker \\ Stefan Grundler Rudolf Maria Huber \\ Division of Pneumology, Department of Internal Medicine, University Hospital, Ludwig Maximilian University, \\ Munich, Germany
}

\section{Key Words}

Functional assay $\cdot$ Isoproterenol $\cdot$ Mucociliary function - Mucus transport velocity · Rotation • Spheroids

\begin{abstract}
Background: We evaluated a new in vitro model for mucociliary transport function. Spheroids of human respiratory epithelium show beating cilia at their surface. When cultured in their own mucus, spheroids can rotate along their axis due to coordinated ciliary beating. Objective and Methods: To assess whether this setup yields meaningful results we measured rotation frequency (RF) of human bronchial or nasal epithelial spheroids under different temperatures and concentrations of isoproterenol. Isoproterenol was administered either as caged compound releasing active isoproterenol after illumination with UV light, or through a permeable membrane in a two-chamber system. Results: Under stable conditions, RF remained constant over $200 \mathrm{~min}$. Between 27 and $35^{\circ} \mathrm{C}$, there was a temperature-dependent increase: $\mathrm{RF}_{27^{\circ} \mathrm{C}}=0.27 \pm 0.08 \mathrm{~s}^{-1}$, and $\mathrm{RF}_{37^{\circ} \mathrm{C}}=0.43 \pm$ $0.10 \mathrm{~s}^{-1}$ (means \pm SEM). Isoproterenol $\left(10^{-5}, 10^{-4}\right.$ and $\left.10^{-3} \mathrm{mmol} / \mathrm{l}\right)$ induced concentration-dependent increases in RF (9, 20 and 25\%, respectively; medians) if applied in the two-chamber system. The experiments with caged isoproterenol did not yield conclusive results, probably
\end{abstract}

because the byproducts from photolysis negatively affected ciliary function. The transport velocity at the surface of bronchial and nasal spheroids was estimated to be 2.96 and $3.62 \mathrm{~mm} / \mathrm{min}$ (medians), respectively, which is in the same range as mucus transport velocity measured in vivo in humans. Conclusions: This setup can be used to study mucociliary transport function under controlled conditions in vitro, in particular as RF is likely to reflect not only ciliary beat frequency, but also the coordination of ciliary beating and the properties of the mucus.

Copyright (C) 2006 S. Karger AG, Basel

\section{Introduction}

The measurement of ciliary beat frequency (CBF) is well introduced for the assessment of mucociliary function. It is commonly performed in isolated patches of cells in the absence of mucus. However, coordination of the ciliary action and the properties of the mucus are not taken into account. Therefore, CBF only partially incorporates the factors affecting epithelial transport capacity in vivo [1]. One alternative approach is the quantification of mucus removal in terms of particle transport velocity in vivo and in vitro.

Cultured spheroids of respiratory epithelium consist of a layer of respiratory epithelial cells which maintain

\section{KARGER \\ Fax +4161306 1234 E-Mail karger@karger.ch} www.karger.com
(C) 2006 S. Karger AG, Basel 0025-7931/06/0735-0664\$23.50/0

Accessible online at: www.karger.com/res
Dr. F. Gamarra

Medizinische Klinik, Ludwig-Maximilians-Universität

Ziemssenstrasse 1

DE-80336 Munich (Germany)

Tel. +49 895160 7534, Fax +49 895160 4905, E-Mail gamarra@med.uni-muenchen.de 


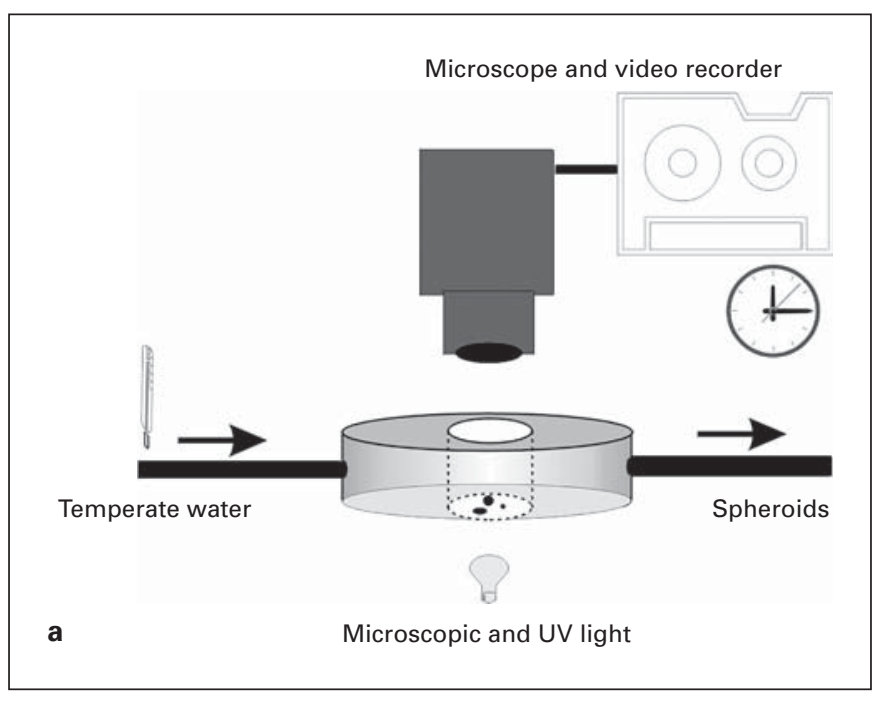

Fig. 1. Schematic diagram of the system to measure RF. a The spheroids were placed into a transparent chamber. Images of the rotating spheroids were acquired through a microscope with a CCD camera and recorded on video. Warmed water flowing through a second lumen permitted to control temperature. Caged isoproterenol was mixed into the medium at the desired concentration and released during the experiments by application of UV light. $\mathbf{b}$ The chamber was modified for a part of the experiments. A cell culture insert $\left(8-\mu \mathrm{m}\right.$ pores, $1.0 \times 10^{8}$ pores $\left./ \mathrm{cm}^{2}\right)$ containing the spheroids,

cell polarity and beating cilia at their outer surface $[2,3]$. Spheroids cultured in their own mucus can rotate along their own axis because of coordinated ciliary beating. We hypothesized that the rotation frequency (RF) reflects the transport capacity of ciliated epithelium.

To evaluate the feasibility of this approach, we assessed in the present study whether RF of human respiratory epithelial spheroids can reliably be quantified. Specifically, human bronchial epithelial spheroids were tested for the effects of time, temperature and different concentrations of isoproterenol. Since mechanical stimulation while adding solutions could theoretically affect the results [4] we compared two methods of applying isoproterenol more gently. The first method was to use 'caged isoproterenol'. Photolabile caged derivatives lack biological activity and can be applied to cells under steady-state conditions without evoking biological responses. The biologically active molecule is then released in the concentration desired following illumination with ultraviolet light. At the same time, a side product (the protecting or caging group) is set free, which should be biologically inactive. In the second method, isoproterenol was delivered through a permeable membrane in a two-chamber system. To determine wheth-

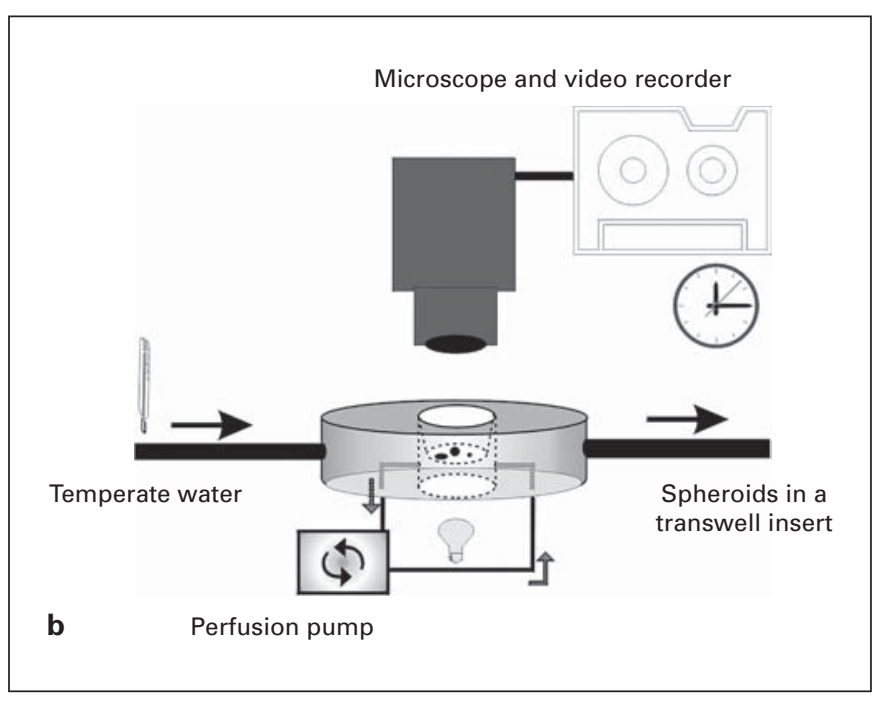

mucus and little medium was placed into the chamber thus creating a second compartment. The lower compartment was filled with medium and connected to a small-volume perfusion pump which permitted to replace the medium with the isoproterenol-containing solution at a defined rate. Isoproterenol molecules diffused through the membrane of the cell culture insert and thus reached the spheroids. This setup permitted to reduce mechanical stimulation of the spheroids during the application of isoproterenol.

er RF of nasal and bronchial spheroids was in a physiologically meaningful range, we also calculated an equivalent of the transport velocity at the surface of the rotating spheroids from their individual RF and diameter.

\section{Materials and Methods}

\section{Harvesting and Culture of Spheroids}

In patients undergoing bronchoscopy for diagnostic or therapeutic reasons, the mucosa of a main bronchus was scraped off with a standard cytology brush to obtain bronchial epithelium. For harvesting human nasal epithelium, the posterior nasal mucosa of healthy, nonsmoking volunteers was scraped off. Patients with HIV or any other acute respiratory infection were excluded. All procedures had been approved by the Institutional Ethical Committee.

The mucus-containing stripes of bronchial or nasal epithelium adhering to the brush were removed by shaking them in a test tube containing bronchial epithelial growth medium (PromoCell, Heidelberg, Germany). The mucus clots were washed two times by carefully pipetting them into Petri dishes filled with medium. The methods were similar to those previously described for organ cultures of respiratory epithelium [5, 6]: Briefly, agar-coated multi-well dishes $(0.75 \% \mathrm{w} / \mathrm{v})$ were prepared according to the following medium formulation: DMEM supplemented with $1,000 \mu \mathrm{g} / \mathrm{ml} \mathrm{glu-}$ cose, 4 times the DMEM-prescribed concentration of nonessential 


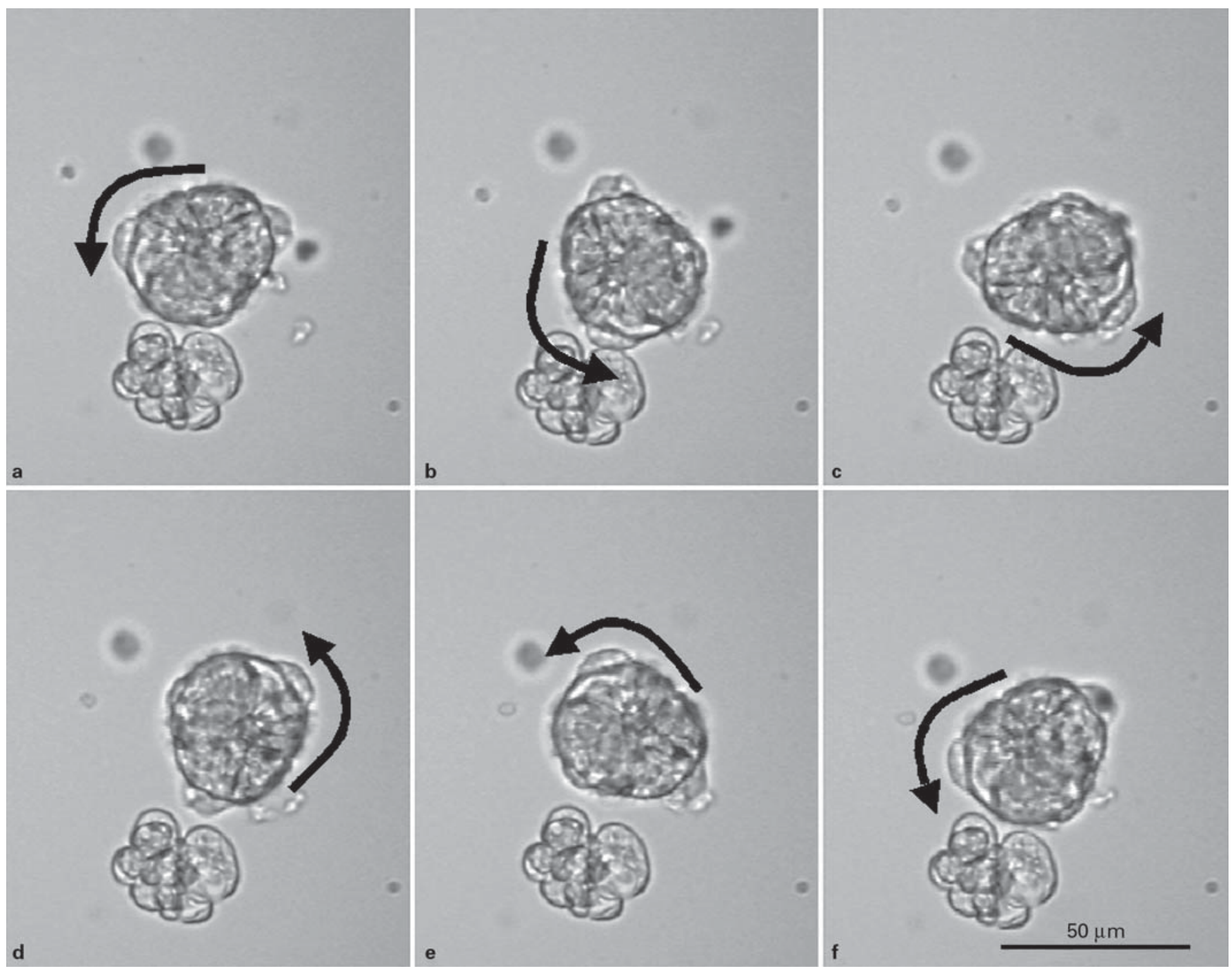

Fig. 2. Repeatedly acquired phase contrast micrographs of a bronchial epithelium spheroid during a complete rotation. While the larger spheroid with cilia at its surface rotates along its own axis, a second nonciliated smaller spheroid does not. The characteristic form of the spheroid permits to quantify RF.

amino acids, $10 \%$ newborn calf serum, penicillin $(10 \mathrm{U} / \mathrm{ml})$, streptomycin $(10 \mu \mathrm{g} / \mathrm{ml})$ and amphotericin B $(1 \mu \mathrm{g} / \mathrm{ml})$; all components were obtained from Life Technologies, Eggenstein, Germany.

The mucus-containing stripes of epithelial cells were then placed into agar-coated wells. Small amounts of bronchial epithelial growth medium containing $10 \%$ newborn calf serum were added in a way that the medium just covered the mucus clots. Cultures were incubated at $37^{\circ} \mathrm{C}, 5 \% \mathrm{CO}_{2}$, with medium changes every 3 days. Rotating spheroids of respiratory epithelium could be identified after 24-48 h of culture using inverse microscopy. Three- to 10-day cultures were used for the experiments.

\section{Measurement $R F$}

Mucus clots with single, rotating spheroids were carefully transferred into a transparent, temperature-controlled chamber (fig. 1a, b) containing $200 \mu \mathrm{l}$ of serum-free, HEPES-buffered DMEM. Images of the rotating spheroids were recorded using a microscope (Stemi 2000; Zeiss, Oberkochen, Germany) equipped with a CCD camera (Hamamatsu Photonics, Hamamatsu City, Japan), video recorder and timer (Panasonic, Matsushita Electric Industrial Co., Osaka, Japan). The frequency of sampling was 30 frames per second. Based on the fact that each spheroid had its particular irregular form which could easily be recognized, the time needed for a full rotation was determined by analyzing the videotapes off-line (fig. 2). For each measurement, three full consecutive rotations were counted and rotation frequency $\left(\mathrm{s}^{-1}\right)$ calculated from the time needed for three rotations. 


\section{Stimulation with Caged Isoproterenol}

Caged isoproterenol [N-(2-nitrobenzyl)isoproterenol; Mobitec, Göttingen, Germany] was dissolved in DMEM with $0.5 \%(\mathrm{v} / \mathrm{v})$ DMSO to a concentration of $10^{-2} \mathrm{mmol} / \mathrm{l}$. DMEM was then added to reach the final concentrations of isoproterenol. During the experiments, the active agent isoproterenol was released by applying UV light ( $\lambda \approx 340 \mathrm{~nm}$ ) for $3 \mathrm{~s}$ (UV-6 SL; Herolab, Wiesloch, Germany) [7].

\section{Stimulation with Isoproterenol Applied in a Two-Compartment} Perfusion Chamber

The above-described temperature-controlled chamber was modified by placing cell culture inserts containing polyethylene terephthalate track-etched, translucent membranes $(8-\mu \mathrm{m}$ pores, $1.0 \times 10^{8}$ pores $/ \mathrm{cm}^{2}$, Falcon ${ }^{\mathrm{TM}}$, Becton-Dickinson, Franklin Lakes, N.J., USA) into the chamber, thereby creating a second compartment (fig. 1b). The rotating spheroids and mucus were positioned with about $50 \mu \mathrm{l}$ of medium in the upper part of the transwell inserts. The lower compartment was connected to a low-volume perfusion pump (Ismatec IPC-N-4, Wetheim-Mohnfeld, Germany) which permitted replacement of the medium at a defined rate. During the experiments, the isoproterenol solution was pumped into the chamber $(400 \mu \mathrm{l}$, about $2 \times$ the volume of the lower compartment, in 1 minute). Isoproterenol thus reached the spheroids by diffusion through the membrane of the cell culture insert.

\section{Experimental Protocols}

Dependency of RF on Time. Bronchial spheroids were incubated at constant temperature $\left(36 \pm 1^{\circ} \mathrm{C}\right)$ for $210 \mathrm{~min}$. RF was measured 7 times during the observation period.

Dependency of $R F$ on Temperature. Bronchial spheroids were exposed to temperatures rising in steps of $2^{\circ} \mathrm{C}$ from 27 to $41^{\circ} \mathrm{C} \mathrm{ev}$ ery $4 \mathrm{~min}$. RF was measured about $3 \mathrm{~min}$ after the last increase in temperature.

Experiments with Caged Isoproterenol. Bronchial spheroids were randomized to incubation with either DMEM or $10^{-5}, 10^{-4}$ or $10^{-3} \mathrm{mmol} / 1$ caged isoproterenol in DMEM. Spheroids were placed into the measurement chamber $\left(36 \pm 1^{\circ} \mathrm{C}\right)$ and given 5 min to adapt to these conditions. After baseline rotation had been assessed over $5 \mathrm{~min}$, UV light was applied and spheroid rotation was recorded for the following $20 \mathrm{~min}$. In order to check for potential artifacts, additional experiments were performed using DMSO and nitrobenzaldehyde for incubation. Nitrobenzaldehyde was used as a substitute of 2-nitrosobenzaldehyde, which is the byproduct of photolysis of caged isoproterenol but not commercially available. DMSO was used as it was necessary for solving the caged isoproterenol in culture medium. Spheroids were incubated with DMEM plus $0.5 \%$ DMSO (v/v), or DMEM plus $10^{-5}, 10^{-4}$ or $10^{-3} \mathrm{mmol} / \mathrm{l}$ nitrobenzaldehyde (containing $0.0005,0.005$ or $0.05 \%$ DMSO, respectively). After $5 \mathrm{~min}$ of adaptation, rotation was documented on video for $20 \mathrm{~min}$. For the experiments above we used spheroids cultured from the bronchial samples of 42 patients. The median age of the donors was 58 years (range 33-86).

Experiments with Isoproterenol Applied in the Two-Compartment Perfusion Chamber. After an adaptation time of $5 \mathrm{~min}$, baseline rotation frequency of bronchial spheroids at $36 \pm 1{ }^{\circ} \mathrm{C}$ was recorded for $5 \mathrm{~min}$. The medium in the lower compartment was then changed by applying isoproterenol in the randomly assigned concentration $\left(0,10^{-5}, 10^{-4}\right.$ or $\left.10^{-3} \mathrm{mmol} / \mathrm{l}\right)$. The rotation of the spheroids was documented for $20 \mathrm{~min}$ after changing the medium.
This experiment was performed with the spheroids of 9 patients (median age 65 years, range 55-79).

Measurements Using Nasal Epithelial Spheroids. Baseline RF was assessed at a constant temperature of $36 \pm 1{ }^{\circ} \mathrm{C}$. A defined magnification was used in order to calculate the size of the spheroids. The spheroids of 18 donors with a median age of 24 years (range 20-50) were used.

\section{Data Analysis and Statistics}

Data are given as means \pm SEM or as median values, depending on data distribution. Changes in RF as a function of time, temperature or isoproterenol were assessed by one-way repeated-measures analysis of variance (ANOVA). Post hoc comparisons were performed by paired t test using appropriate Bonferroni corrections. To compare baseline values in different experimental groups, Kruskal-Wallis one-way ANOVA on ranks or the Mann-Whitney $\mathrm{U}$ test was used. Kinetics of RF in different experimental groups were compared using the two-way repeated-measures ANOVA and the Tukey test. The Pearson product moment correlation was used to assess the dependence of RF on the spheroid size. All analyses were performed with the SigmaStat statistical software package (Jandel Scientific, San Rafael, Calif., USA).

\section{Results}

Spheroids spontaneously formed in $72 \%$ of bronchial samples. Rotating spheroids could be observed in 55\% of the cultures. Nasal brushes yielded spheroids in $94 \%$ of the samples and rotating spheroids developed in $83 \%$.

\section{Baseline RF}

In 141 previously untreated, bronchial epithelium spheroids, baseline RF was $0.42 \pm 0.15 \mathrm{~s}^{-1}$ (mean \pm SEM). In 141 nasal epithelium spheroids, baseline RF was significantly lower: $0.21 \pm 0.12 \mathrm{~s}^{-1}(\mathrm{p}<0.05$, MannWhitney $\mathrm{U}$ test). To assess whether these differences in baseline RF between nasal and bronchial spheroids were related to the fact that nasal samples were taken from healthy, nonsmoking donors whereas bronchial samples had been obtained from patients probably bearing respiratory diseases, we additionally measured RF in 38 bronchial spheroids from a nonsmoking patient with ataxia who did not show evidence of respiratory disease and had been subjected to bronchoscopy solely to exclude the possible presence of a lung tumor. Basal RF in the bronchial spheroids of this donor was $0.25 \pm 0.11 \mathrm{~s}^{-1}$ and thus statistically significantly slower than RF in the bronchial spheroids of the other patients.

\section{Calculated Equivalent Transport Velocity}

Transport velocity at the surface of the spheroids was estimated for the bronchial probes of the patient with 

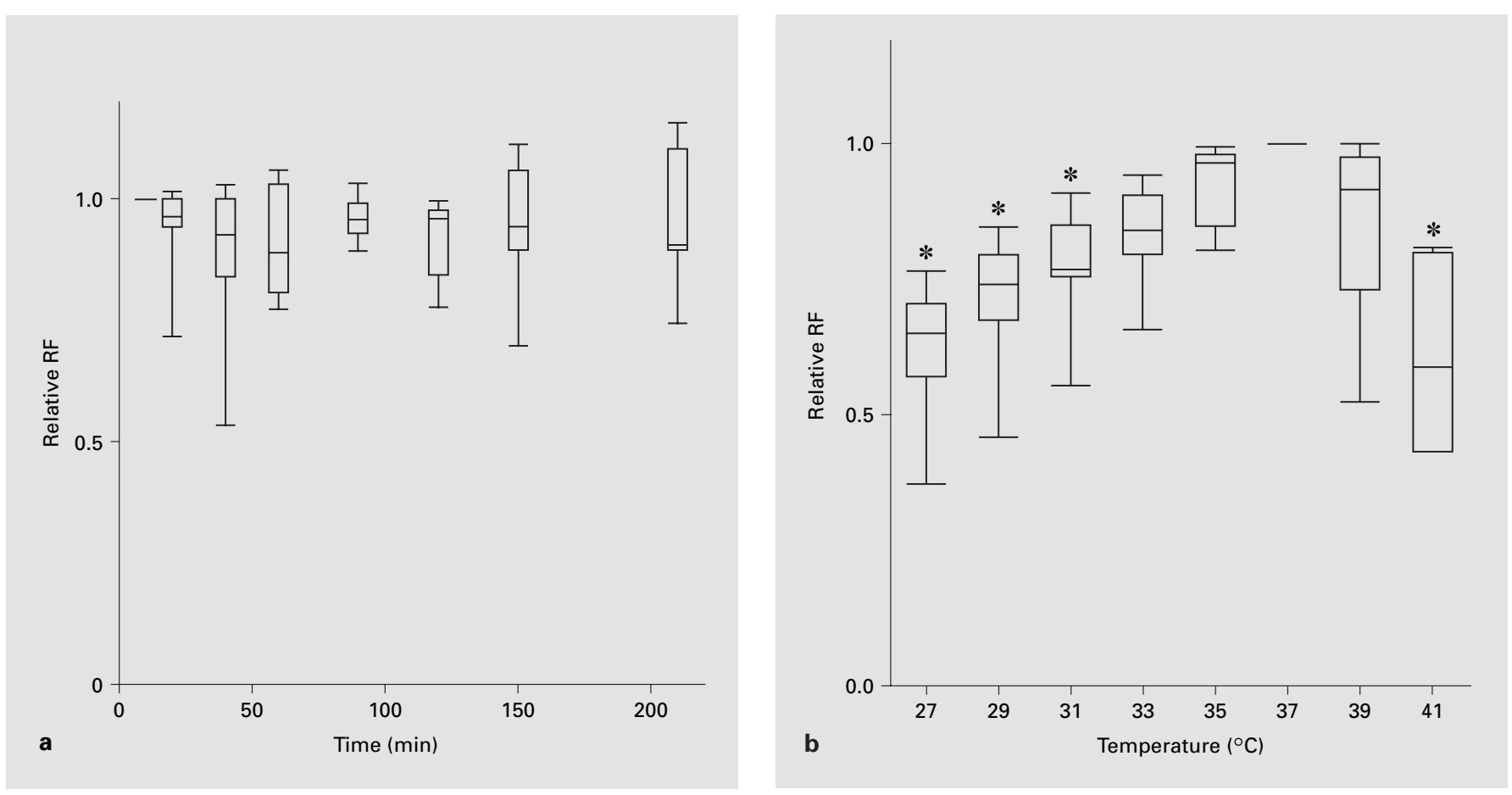

Fig. 3. a Relative changes in RF of bronchial epithelial spheroids during 210 min under stable conditions (box plots showing median, 25th, 75th, 5th and 95th percentiles; $\mathrm{RF}_{10 \min }=1 ; \mathrm{n}=7$ spheroids; $\mathrm{T}=36 \pm 1^{\circ} \mathrm{C}$ ). No significant changes in RF were detected (one-way repeated-measures ANOVA). b Temperature-dependent, relative changes in RF of bronchial epithelium spheroids (box plots showing the median, 25th, 75th, 5th and 95th percentiles; $\mathrm{RF}_{37^{\circ} \mathrm{C}}=1 ; \mathrm{n}=9$ spheroids). $\mathrm{RF}$ significantly increased between 27 and $37^{\circ} \mathrm{C}$ and decreased again at higher temperatures $\left(\mathrm{p}<0.001\right.$, one-way repeated-measures ANOVA; ${ }^{*} \mathrm{p}<0.05 \mathrm{vs} . \mathrm{RF}_{37^{\circ} \mathrm{C}}$ in the Bonferroni $\mathrm{t}$ test).

ataxia and for the nasal spheroids by calculating tangential rotation velocity using the formula $\mathrm{v}=\mathrm{D} \times \pi \times \mathrm{RF}$, whereas $\mathrm{v}$ indicates the transport velocity at the surface and $\mathrm{D}$ the average diameter of the spheroid perpendicular to the rotation axis. Tangential velocity in bronchial spheroids was $3.62 \mathrm{~mm} / \mathrm{min}$ (median), whereas in nasal spheroids it was $2.96 \mathrm{~mm} / \mathrm{min}$ and thus significantly slower ( $p<0.05$, Mann-Whitney U test). Bronchial spheroids of the ataxia patient had a median diameter of $80 \mu \mathrm{m}$ (median); the diameter of nasal spheroids was $90 \mu \mathrm{m}$ (nonsignificant, Mann-Whitney U test). Furthermore, in nasal spheroids there was a weak but statistically significant inverse correlation between RF and the diameter of the spheroids (correlation coefficient = $-0.182 ; \mathrm{p}<0.05$, Pearson product moment correlation), consistent with the assumption that at constant transport velocity RF and diameter should be inversely proportional to each other. This relationship was not apparent in the bronchial spheroids.

\section{Stability of RF over Time}

RF of bronchial spheroids seemed to remain constant over 200 min under stable conditions (fig. 3a). In the oneway repeated-measures ANOVA, no statistically significant differences were detected. The power of the test was however low (0.050), so that the findings have to be interpreted cautiously.

\section{Effects of Temperature on $R F$}

In bronchial spheroids, an increase in temperature from 27 to $37^{\circ} \mathrm{C}$ induced a gradual, statistically significant increase in RF ( $p<0.001$, one-way repeated-measures ANOVA). At $27^{\circ} \mathrm{C}$, RF was $0.27 \pm 0.08 \mathrm{~s}^{-1}$ (mean $\pm \mathrm{SEM}$ ), whereas it was $0.43 \pm 0.10 \mathrm{~s}^{-1}$ at $37^{\circ} \mathrm{C}$. The corresponding relative changes are shown in figure $3 \mathrm{~b}$. When temperature was further raised to 39 and $41^{\circ} \mathrm{C}, \mathrm{RF}$ decreased and some spheroids even stopped. Additional experiments showed that if temperature was set from 37 to $27^{\circ} \mathrm{C}$ and raised again, the corresponding decrease in $\mathrm{RF}$ was reversible. However, when temperature was in- 
creased to $39^{\circ} \mathrm{C}$ and higher, changes in RF were partially irreversible (data not shown).

\section{Effects of Isoproterenol on RF}

In order to test the effect of isoproterenol, two different methods were used for the application of the compound. In both experiments, baseline RF did not significantly differ between the groups (Kruskal-Wallis one-way ANOVA on ranks). In the experiments with caged isoproterenol (fig. 4a), illumination with UV light induced an immediate, statistically significant increase in RF in the groups exposed to caged isoproterenol but not in the control group ( $\mathrm{p}<0.05$ for $10^{-5} \mathrm{mmol} / \mathrm{l}$ and $\mathrm{p}<0.01$ for $10^{-4}$ and $10^{-3} \mathrm{mmol} / 1$ caged isoproterenol at $\mathrm{t}=2 \mathrm{~min}$; two-way repeated-measures ANOVA and Tukey test). It seems reasonable to attribute this increase to the release of isoproterenol by photolysis. The maximum effect was found for $10^{-4} \mathrm{mmol} / \mathrm{l}$ isoproterenol. After the initial increase, RF decreased again in the groups exposed to caged isoproterenol, especially at the highest concentration $\left(10^{-3} \mathrm{mmol} / \mathrm{l}\right)$. RF was constant over time in the spheroids that were exposed to medium only. When spheroids were exposed to DMSO or nitrobenzaldehyde RF continuously decreased (fig. 4b). Compared to baseline, significantly lower RF values were measured at $t=$ 16 and $18 \mathrm{~min}$ for DMSO $(\mathrm{p}<0.05)$, at $\mathrm{t}=12 \mathrm{~min}$ for $10^{-5} \mathrm{mmol} / 1$ nitrobenzaldehyde $(\mathrm{p}<0.01)$ and at $\mathrm{t} \geq$ $8 \mathrm{~min}$ for $10^{-4}$ and $10^{-3} \mathrm{mmol} / 1$ nitrobenzaldehyde $(\mathrm{p}<$ 0.001 ). The groups exposed to $10^{-4}$ and $10^{-3} \mathrm{mmol} / \mathrm{l} \mathrm{ni-}$ trobenzaldehyde differed significantly from the medium control $(\mathrm{p}<0.01)$.

When isoproterenol was applied via cell culture insert membranes (fig. 4c), there was an initial slight reduction followed by statistically significant RF increases of 20 and $25 \%$ (median values) in the spheroids exposed to $10^{-4}$ and $10^{-3} \mathrm{mmol} / 1$ isoproterenol, respectively $(\mathrm{p}<0.001$ for $\mathrm{t}=$ $2 \mathrm{~min}$ to $\mathrm{t}=5 \mathrm{~min}$ vs. baseline in both groups, Tukey test). In the group treated with $10^{-5} \mathrm{mmol} / 1$ isoproterenol, the increase in RF was less pronounced ( $9 \%$, median). Even smaller and nonsignificant changes in RF were seen in the medium control group. The effect was not only more pronounced but also lasted longer at the highest isoproterenol concentration. These results suggest a dose-dependent effect of isoproterenol on RF, but the differences between the effects of different isoproterenol concentrations were small and did not reach statistical significance (two-way repeated-measures ANOVA).

Rotation Frequency of Bronchial and Nasal Epithelial Spheroids

\section{Discussion}

In the present study, we measured RF of human bronchial and nasal epithelial spheroids in vitro. The rationale for these experiments was the assumption that spheroid rotation by coordinated ciliary beating might be a comprehensive measure of epithelial transport capacity. Under the conditions of measurement, RF remained stable for more than $3 \mathrm{~h}$ and was dependent on temperature, whereby the values almost doubled between 27 and $37^{\circ} \mathrm{C}$, consistent with the temperature dependence of biochemical and cellular processes. An acute increase in RF induced by isoproterenol was detectable, as already known for CBF, which suggests that RF is in fact a function of ciliary beating activity. The calculated equivalent transport velocity at the spheroid surface was about 3-4 $\mathrm{mm} /$ min and thus of the same order of magnitude as particle transport velocity values measured in vivo.

Cultured spheroids of respiratory epithelium bearing cilia have been first described by Corssen and Allen [2] and more recently by Bridges et al. [8] and Jorissen et al. [3]. In the nose of normal healthy humans, mucus transport velocity (measured scintigraphically using technetium-labeled macroaggregated human albumin) has been reported to be about $8 \mathrm{~mm} / \mathrm{min}$ [9], whereas tracheal mucus velocities were about $11 \mathrm{~mm} / \mathrm{min}$ [10]. The values we calculated for transport velocity at the surface of cultured spheroids are in the same range, but 2-3 times lower compared to those measured in vivo. One possible explanation is that the in vivo techniques require the deposition of labeled particles on the mucosa, a procedure which could already act as a stimulus to the cilia. As a consequence, transport velocity might be overestimated using particle deposition techniques in vivo [11]. In our experiments, nasal spheroids had been obtained from healthy, young nonsmokers. The RF and the calculated equivalent transport velocity at the surface of nasal spheroids were lower as those observed in bronchial samples from 1 nonsmoking patient without apparent respiratory disease, as would be expected from the published data. Although CBF is one of the major factors of mucus transport, other factors such as the orientation and coordination of ciliary beating, the viscous properties of the mucus and the interaction between cilia and mucus are also involved. The measurement of $\mathrm{CBF}$ alone is therefore not sufficient to predict the effects of interventions on mucus transport velocity [1]. Cultured spheroids of respiratory epithelium, as presented here, combine advantages of a classical cell culture system (i.e. standardized conditions) with the possibility to approach the complexities of mucus transport as close as possible.

Respiration 2006;73:664-672 
Fig. 4. a Relative changes in RF in spheroids exposed to medium alone or to different concentrations of caged isoproterenol prior to and after release of isoproterenol following irradiation with UV light. The first values, before the application of UV light, were normalized to 1 (median values; $\mathrm{n}=10$ spheroids/group). Statistically significant increases in RF were measured in the groups exposed to caged isoproterenol $(\mathrm{p}<$ 0.05 for $10^{-5} \mathrm{mmol} / \mathrm{l}$ and $\mathrm{p}<0.01$ for $10^{-4}$ and $10^{-3} \mathrm{mmol} / \mathrm{l}$ caged isoproterenol at $\mathrm{t}=$ 2 min; two-way repeated-measures ANOVA and Tukey test). After the initial increase, RF values decreased again, especially in the $10^{-3} \mathrm{mmol} / \mathrm{l}$ caged isoproterenol group. In the medium control group, RF remained constant over time. The differences between the experimental groups did not reach statistical significance. b Relative changes in RF in spheroids exposed to medium, $0.5 \%$ DMSO or increasing concentrations of nitrobenzaldehyde. The first values were normalized to 1 (median values; $\mathrm{n}=10$ spheroids in the DMEM group, $\mathrm{n}=$ 5 spheroids in the DMSO and nitrobenzaldehyde groups). RF decreased significantly in the groups exposed to DMSO and to the different concentrations of nitrobenzaldehyde $(\mathrm{p}<0.05$ at $\mathrm{t}=16$ and $18 \mathrm{~min}$ for DMSO; $\mathrm{p}<0.05$ at $\mathrm{t}=12 \mathrm{~min}$ for $10^{-5} \mathrm{mmol} / 1$ nitrobenzaldehyde; $\mathrm{p}<0.001$ at $\mathrm{t} \geq 8 \mathrm{~min}$ for $10^{-4}$ and $10^{-3} \mathrm{mmol} / 1$ nitrobenzaldehyde; two-way repeated-measures ANOVA and Tukey test). There were also statistically significant differences between the $10^{-4}$ and $10^{-3} \mathrm{mmol} / 1$ nitrobenzaldehyde groups and the medium control $(\mathrm{p}<$ $0.01)$.
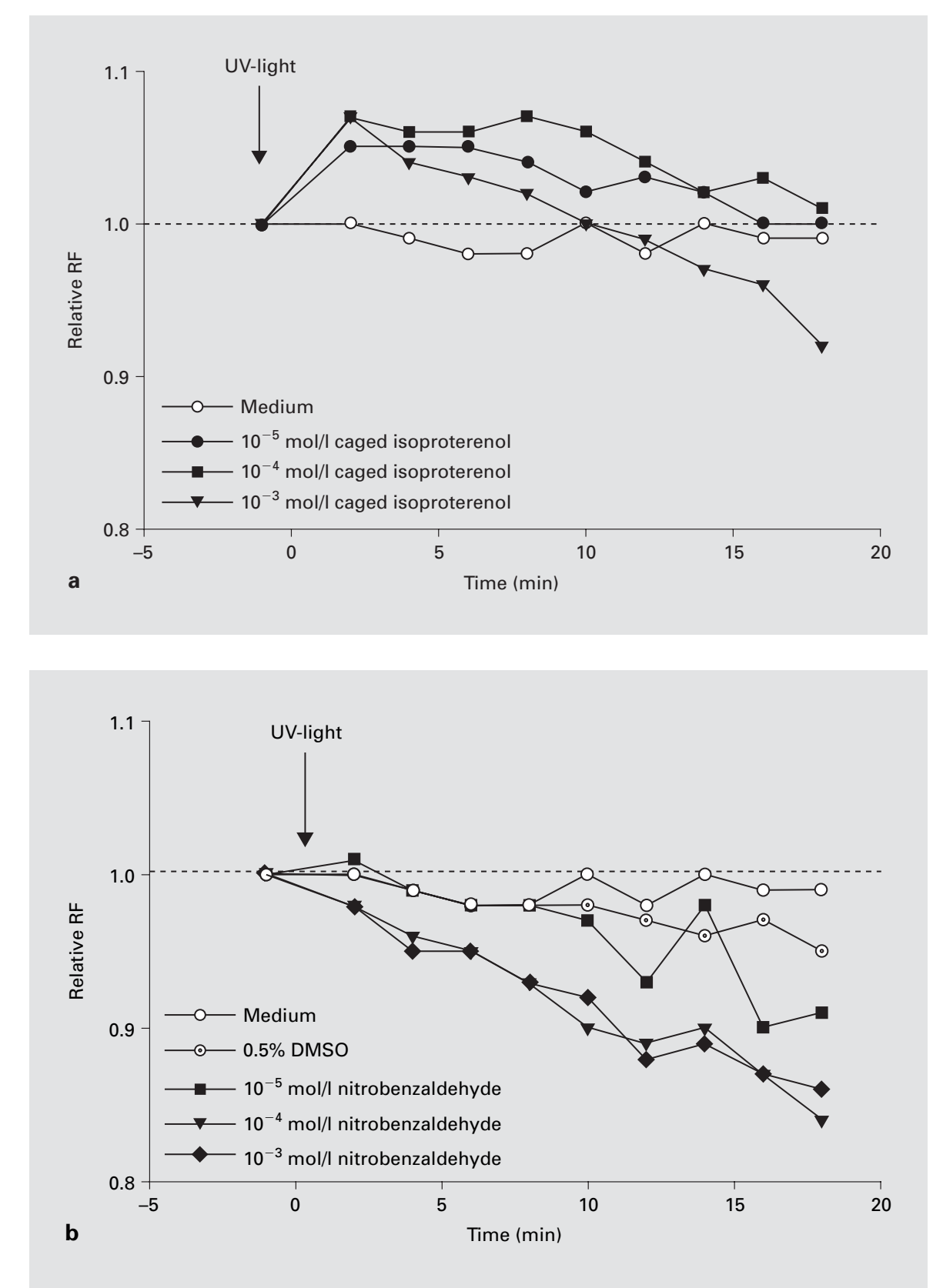

One major disadvantage of the spheroid system is the fact that at present we are neither able to control for the spheroid size nor for the properties of the mucus. In addition, it is not possible to exclude unwanted donor-dependent properties of the mucus or the form of the spheroids which might affect rotation. The variability in RF values of different spheroids is quite high.

Assuming that the beating of cilia at the spheroid surface produces a constant tangential velocity, RF, i.e. the reciprocal of the time period needed for one complete rotation, should be inversely proportional to the average diameter. There was a weak relationship between RF and the size of nasal spheroids only. Other factors like a reduced efficiency of movement because of a low number of cilia involved, or a greater viscous drag at the surface relative to the force exerted by the cilia in smaller spheroids have to be considered. 
Fig. 4. c Relative RF in bronchial epithelium spheroids after application of medium or different concentrations of isoproterenol through a permeable membrane. RF values at $\mathrm{t}=-0.5 \mathrm{~min}$, prior to the beginning of medium exchange, were normalized to 1 (median values; $\mathrm{n} \geq 12$ spheroids/group). During medium exchange, RF decreased slightly, probably due to a transient reduction in temperature in the chamber. Subsequently, there was a statistically significant increase in RF in the groups exposed to $10^{-4}$ and $10^{-3} \mathrm{mmol} / 1$ isoproterenol ( $p<0.001$ for $\mathrm{t}=2 \mathrm{~min}$ to $\mathrm{t}=5 \mathrm{~min}$ vs. baseline in both groups, two-way repeatedmeasures ANOVA and Tukey test). In the $10^{-5} \mathrm{mmol} / 1$ isoproterenol group and the control group, the changes were less pronounced and not statistically significant. The differences between the single groups did not reach statistical significance (twoway repeated-measures ANOVA).

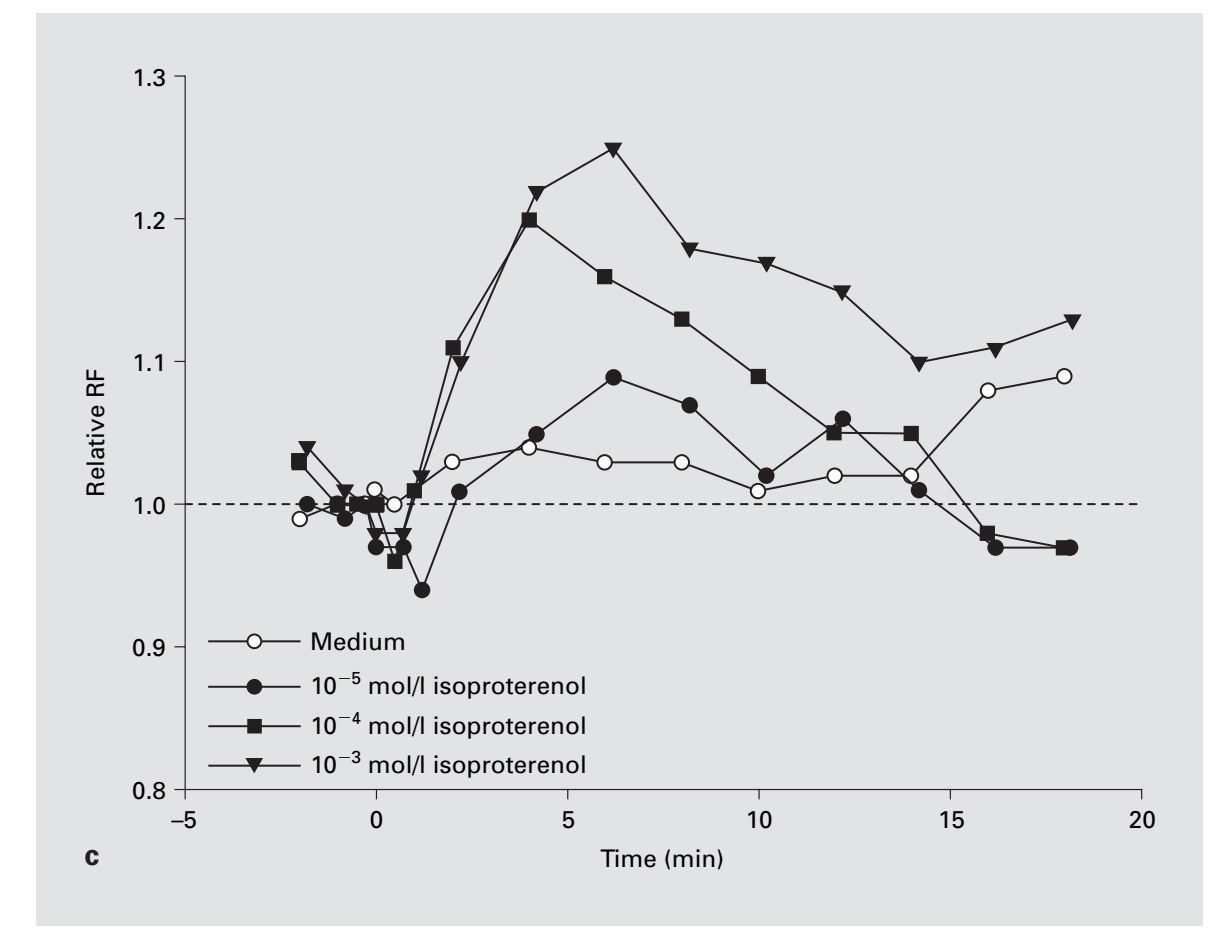

It has been shown that there is a linear rise in $\mathrm{CBF}$ as a function of temperature between 20 and $40^{\circ} \mathrm{C}$. At 40 $44^{\circ} \mathrm{C} \mathrm{CBF}$ remains constant whereas it decreases at higher temperatures, probably because of hyperthermic cell damage [12-15]. More recent measurements of CBF in relation to mucus transport yielded similar results [16]. The rise in RF observed in our experiments between 27 and $37^{\circ} \mathrm{C}$ corresponded well to these findings. At temperatures of 39 and $41^{\circ} \mathrm{C}$, we observed a reduction in $\mathrm{RF}$ that was not expected from the data on CBF. It might be that a decrease in viscosity of the surrounding mucus beginning at $39^{\circ} \mathrm{C}$ led to a loss of coupling of ciliary beat to the medium and thus to a reduction in the rotation rate. Another possible explanation might be that the signaling between cells, which mediates the coordination of cilia, was already affected at these temperatures.

To test the sensitivity of the spheroid system to pharmacological stimuli, we used different concentrations of isoproterenol. In human nasal epithelial cell cultures exposed to $10^{-5}$ and $10^{-3} \mathrm{mmol} / \mathrm{l}$ isoproterenol, $\mathrm{CBF}$ increases of about $9-17 \%$ have been demonstrated [17, 18]. Kurosawa et al. [13] studied the isoproterenol-induced changes in $\mathrm{CBF}$ and particle transport velocity in explanted lung tissue of mice. Both measures increased in a concentration-dependent manner, showing a plateau between $10^{-5}$ and $10^{-2} \mathrm{mmol} / \mathrm{l}$, thus suggesting that at con- centrations higher than $10^{-5} \mathrm{mmol} / 1$ the maximal effect of isoproterenol on CBF is reached. The effects started $1 \mathrm{~min}$ after administration of isoproterenol and lasted for at least $5 \mathrm{~min}$. In our experiments in the two-compartment chamber, we observed an immediate reduction in $\mathrm{RF}$ after application of isoproterenol, probably as a consequence of a transient decrease in temperature during the medium exchange in the chamber. The subsequent increase in RF seemed to depend on the concentration of isoproterenol, although relatively high concentrations were used. The RF responses were smaller and of shorter duration as those described for CBF and showed an increased variability.

We hypothesized that caged molecules would provide an elegant method for pharmacological interventions on rotating spheroids, as they allowed to avoid temperature effects and mechanical stimulation, which could per se alter RF [4, 19]. The experiments performed showed however that the effects of DMSO, which was used as solving agent, as well as the byproducts of caged molecules, which were released after photolysis, also had to be considered. $\mathrm{N}$-(2-nitrobenzyl)isoproterenol has been shown to retain some agonist activity (10-20\% that of isoproterenol), as measured via the relaxation of isolated rat mesenteric arterioles [20]. This residual $\beta$-agonist activity of caged isoproterenol might have increased base- 
line values of RF in our experiments. Indeed, these were slightly though not statistically significantly elevated compared to the control group treated with the culture medium DMEM. Unfortunately, photolysis by UV light produced a byproduct of caged isoproterenol, which is 2nitrosobenzaldehyde [21]. This does not seem to have measurable effects on the diameter of rat mesenteric arterioles [20]. The rapid decrease in RF after the first stimulatory effect of the photoreleased isoproterenol suggested that the byproduct of the procedure might have detrimental effects on RF. As 2-nitrosobenzaldehyde is not commercially available as a stable substance, we chose nitrobenzaldehyde dissolved in DMSO as a substitute with similar chemical structure. Indeed, nitrobenzaldehyde induced a steady decrease in RF in a concentrationdependent manner. These data indicate toxic effects and thus appear to render the use of caged isoproterenol unsuitable for the study of epithelial spheroids.

In conclusion, the data of the present study indicate that the measurement of RF of cultured nasal or bronchial epithelial spheroids is feasible in vitro and that this model shows a number of features that are in agreement with the characteristics of ciliary beating or mucociliary transport found in vivo or in vitro. As the spheroid system involves a higher level of cellular complexity than the study of single ciliated cells, while still being not more difficult to handle experimentally, it might be particularly useful for studies involving the coordination of ciliary beating and the relation of $\mathrm{CBF}$ to the effective transport velocity. Rotational velocity, as an easy-to-measure putative equivalent of mucus transport velocity in ciliated epithelium, seems a promising tool to be evaluated in future studies.

\section{Acknowledgments}

This study was in part supported by the Wilhelm Sander and the Friedrich Baur foundations. We thank Dr. R.A. Jorres, Institute of Occupational and Environmental Medicine, Ludwig Maximilians University, Munich, Germany, for kindly revising the manuscript.

\section{References}

1 Jorissen M: Correlations among mucociliary transport, ciliary function, and ciliary structure. Am J Rhinol 1998; 12:53-58.

2 Corssen G, Allen CR: Acetylcholine: its significance in controlling ciliary activity of human respiratory epithelium in vitro. J Appl Physiol 1959;14:901-904.

3 Jorissen M, Van der SB, Tyberghein J, Van der $\mathrm{BH}$, Cassiman JJ: Ciliogenesis and coordinated ciliary beating in human nasal epithelial cells cultured in vitro. Acta Otorhinolaryngol Belg 1989;43:67-73.

4 Sanderson MJ, Dirksen ER: Mechanosensitivity of cultured ciliated cells from the mammalian respiratory tract: implications for the regulation of mucociliary transport. Proc Natl Acad Sci USA 1986;83:7302-7306.

5 Al Batran SE, Astner ST, Supthut M, Gamarra F, Brueckner K, Welsch U, Knuechel R, Huber $\mathrm{RM}$ : Three-dimensional in vitro cocultivation of lung carcinoma cells with human bronchial organ culture as a model for bronchial carcinoma. Am J Respir Cell Mol Biol 1999;21: 200-208.

6 Gamarra F, Wagner S, Al Batran S, Maier I, Castro M, Hautmann H, Bergner A, Baumgartner R, Huber RM: Kinetics of 5-aminolevulinic acid-induced fluorescence in organ cultures of bronchial epithelium and tumor. Respiration 2002;69:445-450.

7 Adams SR, Tsien RY: Controlling cell chemistry with caged compounds. Annu Rev Physiol 1993;55:755-784.
8 Bridges MA, Walker DC, Harris RA, Wilson BR, Davidson AG: Cultured human nasal epithelial multicellular spheroids: polar cyst-like model tissues. Biochem Cell Biol 1991;69: 102-108.

9 Armengot M, Basterra J, Garcia-Bartual E: The influence of anesthetics and vasoconstrictors on nasal mucociliary transport. Acta Otorhinolaryngol Belg 1989;43:149-156.

10 Morgan L, Pearson M, de Iongh R, Mackey D, van der Wall H, Peters M, Rutland J: Scintigraphic measurement of tracheal mucus velocity in vivo. Eur Respir J 2004;23:518-522.

11 Wanner A, Salathe M, O'Riordan TG: Mucociliary clearance in the airways. Am J Respir Crit Care Med 1996;154:1868-1902.

12 Jorissen M, Bessems A: Influence of culture duration and ciliogenesis on the relationship between ciliary beat frequency and temperature in nasal epithelial cells. Eur Arch Otorhinolaryngol 1995;252:451-454.

13 Kurosawa H, Wang CG, Dandurand RJ, King M, Eidelman DH: Mucociliary function in the mouse measured in explanted lung tissue. $\mathbf{J}$ Appl Physiol 1995;79:41-46.

14 Mercke U, Hakansson $\mathrm{CH}$, Toremalm NG: The influence of temperature on mucociliary activity. Temperature range 20 degrees C-40 degrees C. Acta Otolaryngol 1974;78:444450 .
15 Mercke U: The influence of temperature on mucociliary activity. Temperature range 40 degrees $\mathrm{C}$ to 50 degrees $\mathrm{C}$. Acta Otolaryngol 1974;78:253-258.

16 Kilgour E, Rankin N, Ryan S, Pack R: Mucociliary function deteriorates in the clinical range of inspired air temperature and humidity. Intensive Care Med 2004;30:1491-1494.

17 Agu RU, Jorissen M, Willems T, Van den Mooter G, Kinget R, Augustijns P: Effects of pharmaceutical compounds on ciliary beating in human nasal epithelial cells: a comparative study of cell culture models. Pharm Res 1999; 16:1380-1385.

18 Wyatt TA, Spurzem JR, May K, Sisson JH: Regulation of ciliary beat frequency by both PKA and PKG in bovine airway epithelial cells. Am J Physiol 1998;275:L827-L835.

19 Sanderson MJ, Dirksen ER: Mechanosensitive and beta-adrenergic control of the ciliary beat frequency of mammalian respiratory tract cells in culture. Am Rev Respir Dis 1989;139:432440 .

20 Muralidharan S, Nerbonne JM: Photolabile 'caged' adrenergic receptor agonists and related model compounds. J Photochem Photobiol B 1995;27:123-137.

21 Muralidharan S, Maher GM, Boyle WA, Nerbonne JM: 'Caged' phenylephrine: development and application to probe the mechanism of alpha-receptor-mediated vasoconstriction. Proc Natl Acad Sci USA 1993;90:5199-5203. 